\title{
Design of Reversible Sequential Circuit Using Reversible Logic Synthesis
}

\author{
Md. Belayet Ali ${ }^{1}$, Md. Mosharof Hossin ${ }^{1}$ and Md. Eneyat Ullah ${ }^{1}$ \\ ${ }^{1}$ Department of Computer Science and Engineering \\ Mawlana Bhashani Science and Technology University, Santosh, Tangail-1902, \\ Bangladesh \\ belayet.mbstulgmail.com, mosharof_cselyahoo.com, eneyat_mbstulyahoo.com
}

\begin{abstract}
Reversible logic is one of the most vital issue at present time and it has different areas for its application, those are low power CMOS, quantum computing, nanotechnology, cryptography, optical computing, DNA computing, digital signal processing (DSP), quantum dot cellular automata, communication, computer graphics. It is not possible to realize quantum computing without implementation of reversible logic. The main purposes of designing reversible logic are to decrease quantum cost, depth of the circuits and the number of garbage outputs. In this paper, we have proposed a new reversible gate. And we have designed $R S$ flip flop and D flip flop by using our proposed gate and Peres gate. The proposed designs are better than the existing proposed ones in terms of number of reversible gates and garbage outputs. So, this realization is more efficient and less costly than other realizations.
\end{abstract}

\section{Keywords}

Reversible logic, Reversible gate, Power dissipation, Flip-Flop, Garbage, BME gate.

\section{Introduction}

Energy dissipation is an important consideration in VLSI design. Reversible logic was first related to energy when Landauer states that information loss due to function irreversibility leads to energy dissipation[1].This principle is further supported by Bennett that zero energy dissipation can be achieved only when the circuit contains reversible gates [2].Information is lost when the input vector cannot be uniquely recovered from its output vectors. Reversible logic circuits naturally take care of heating since in a reversible logic every input vector can be uniquely recovered from its output vectors and therefore no information is lost. According to [2] zero energy dissipation would be possible only if the network consists of reversible gates. Thus reversibility will become an essential property in future circuit design. Reversible circuits are also interesting because the loss of bits of information implies energy loss [2]. Younis and Knight [3] showed that some reversible circuits can be made asymptotically energy-lossless if their delay is allowed to be arbitrarily large. However, reversible logic is suffering from two problems. Firstly, there is a lack of technologies with which to build reversible gates. Work is certainly continuing in this area. Secondly, while there is much research into how to design combinational circuits

DOI : $10.5121 /$ vlsic.2011.2404 
using reversible logic, there is little in the area of sequential reversible logic implementations. There is no limitation inherent to reversible logic preventing the design of sequential circuits; in fact when Tommaso Toffoli first characterized reversible logic in his 1980 work Reversible Computing [4] he stated that "Using invertible logic gates, it is ideally possible to build a sequential computer with zero internal power dissipation. "To establish the relevance of reversible and quantum computing it seems appropriate to note that the VLSI industry is moving at high speed towards miniaturization. With miniaturization it faces two issues: i) A considerable amount of energy gets dissipated in VLSI circuits and ii) the size of the transistors are approaching the quantum limits where tunneling and other quantum phenomena are likely to appear. Thus, we need a superior technology that can circumvent these problems.

This paper presents a new $4 * 4$ reversible logic gate that is BME gate. BME gate is a universal in the sense that it can be used to synthesize any arbitrary Boolean function. It is shown that a RS and D Flip-Flop can be realized using proposed reversible logic gate and Peres gate. The presented design reduces the number of gates and the number of garbage outputs.

\section{Reversible Logic Synthesis and Others}

\section{Definition 2.1}

A circuit is reversible if it maps each input vector into a unique output vector and vice versa.

\section{Definition 2.2}

Reversible Gates are circuits in which number of outputs is equal to the number of inputs and there is a one to one correspondence between the vector of inputs and outputs [5], [10] and [12].

Example 2.1. Let the input vector be $I_{v}$, output vector $\mathrm{O}_{v}$ and they are defined as follows, $\mathrm{I}_{\mathrm{v}}=\left(\mathrm{I}_{\mathrm{i}}, \mathrm{I}_{\mathrm{i}+1}, \mathrm{I}_{\mathrm{i}+2} \ldots \mathrm{I}_{\mathrm{k}-1}, \mathrm{I}_{\mathrm{k}}\right)$ and $\mathrm{O}_{\mathrm{v}}=\left(\mathrm{O}_{\mathrm{i}}, \mathrm{O}_{\mathrm{i}+1}, \mathrm{O}_{\mathrm{i}+2} \ldots \mathrm{O}_{\mathrm{k}-1}, \mathrm{O}_{\mathrm{k}}\right)$. For each particular $\mathrm{i}$, there exits the relationship $\mathrm{I}_{\mathrm{v}} \leftrightarrow \square \mathrm{O}_{\mathrm{v}}[10]$.

A reversible circuit should be designed using minimum number of reversible logic gates. From the point of view of reversible circuit design, there are many parameters for determining the complexity and performance of circuits [6], [7], [8] and [11].

\section{Definition 2.3}

Garbage output refers to the number of inputs that are to be maintained constant at either 0 or 1 in order to synthesize the given logical function [12].

\section{Definition 2.4}

Quantum cost refers to the cost of the circuit in terms of the cost of a primitive gate. It is calculated knowing the number of primitive reversible logic gates $(1 * 1$ or $2 * 2)$ required to realize the circuit. The quantum cost of a circuit is the minimum number of $2 * 2$ unitary gates to represent the circuit keeping the output unchanged. The quantum cost of a $1 * 1$ gate is 0 and that of any $2 * 2$ gate is the same, which is 1 [19]. So, the quantum cost of a $2 * 2$ Feynman gate is 1 . Again, the quantum cost of a $3 * 3$ Toffoli, Fredkin, Peres and New gate is 5, 5, 4 and 11 respectively [20][21][22]. 
International Journal of VLSI design \& Communication Systems (VLSICS) Vol.2, No.4, December 2011

\section{Definition 2.5}

Flexibility refers to the universality of a reversible logic gate in realizing more functions [12].

\section{Definition 2.6}

Gate Level refers to the number of levels in the circuit which are required to realize the given logic functions [12].

\section{Definition 2.7}

Hardware Complexity refers to the total number of logic operation in a circuit. Means the total number of AND, OR and EXOR operation in a circuit [9] and [12].

\section{Major Several Reversible Logic}

\subsection{Feynman Gate}

It is a $2 * 2$ Feynman gate [13]. The input vector is $\mathrm{I}(\mathrm{A}, \mathrm{B})$ and the output vector is $\mathrm{O}(\mathrm{P}, \mathrm{Q})$. The outputs are defined by $\mathrm{P}=\mathrm{A}, \mathrm{Q}=\mathrm{A} \oplus \mathrm{B}$. Quantum cost of a Feynman gate is 1 . Figure 1 shows a $2 * 2$ Feynman gate.

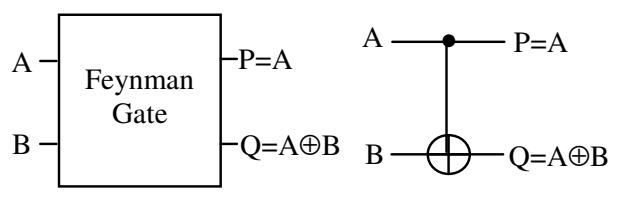

Figure 1: Feynman gate

\subsection{Double Feynman Gate (F2G)}

It is a $3 * 3$ Double Feynman gate [14].The input vector is I (A, B, C) and the output vector is O $(\mathrm{P}, \mathrm{Q}, \mathrm{R})$. The outputs are defined by $\mathrm{P}=\mathrm{A}, \mathrm{Q}=\mathrm{A} \oplus \mathrm{B}, \mathrm{R}=\mathrm{A} \oplus \mathrm{C}$. Quantum cost of double Feynman gate is 2 .

Figure 2 shows a $3 * 3$ Double Feynman gate.

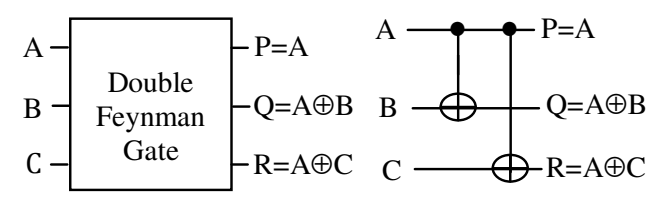

\subsection{Toffoli Gate}

Figure 2: Double Feynman gate

It is a $3 * 3$ Toffoli gate [6] The input vector is $I(A, B, C)$ and the output vector is $O(P, Q, R)$. The outputs are defined by $\mathrm{P}=\mathrm{A}, \mathrm{Q}=\mathrm{B}, \mathrm{R}=\mathrm{AB} \oplus \mathrm{C}$. Quantum cost of a Toffoli gate is 5 . Figure 3 shows a $3 * 3$ Toffoli gate. 
International Journal of VLSI design \& Communication Systems (VLSICS) Vol.2, No.4, December 2011
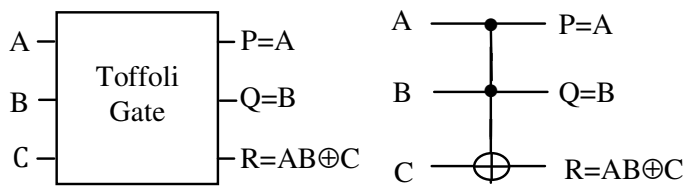

Figure 3: Toffoli gate

\subsection{Fredkin Gate}

It is a $3 * 3$ Fredkin gate [7]. The input vector is $I(A, B, C)$ and the output vector is $O(P, Q, R)$. The output is defined by $\mathrm{P}=\mathrm{A}, \mathrm{Q}=\overline{\mathrm{A}} \mathrm{B} \oplus \mathrm{AC}$ and $\mathrm{R}=\overline{\mathrm{A}} \mathrm{C} \oplus \mathrm{AB}$. Quantum cost of a Fredkin gate is 5. Figure 4 shows a $3 * 3$ Fredkin gate.

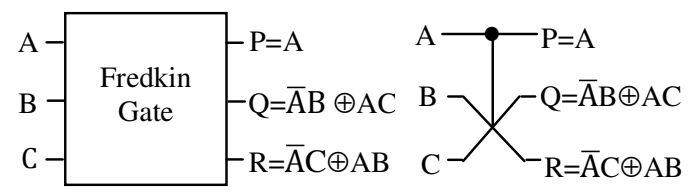

Figure 4: Fredkin gate

\subsection{Peres Gate}

It is a $3 * 3$ Peres gate [15]. The input vector is $I(A, B, C)$ and the output vector is $O(P, Q, R)$. The output is defined by $\mathrm{P}=\mathrm{A}, \mathrm{Q}=\mathrm{A} \oplus \mathrm{B}$ and $\mathrm{R}=\mathrm{AB} \oplus \mathrm{C}$. Quantum cost of a Peres gate is 4 . Figure 5 shows a $3 * 3$ Peres gate.
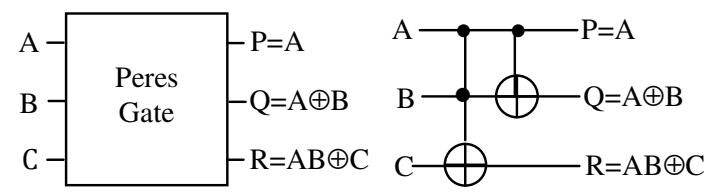

Figure 5: Peres gates

\subsection{Double Peres gate}

It is a $4 * 4$ Double Peres Gate [16]. The input vector is $I(A, B, C, D)$ and the output vector is $\mathrm{O}(\mathrm{P}, \mathrm{Q}, \mathrm{R}, \mathrm{S})$. The output is defined by $\mathrm{P}=\mathrm{A}, \mathrm{Q}=\mathrm{A} \oplus \mathrm{B}, \mathrm{R}=\mathrm{A} \oplus \mathrm{B} \oplus \mathrm{D}$ and $\mathrm{S}=(\mathrm{A} \oplus \mathrm{B}) \mathrm{D} \oplus \mathrm{AB} \oplus \mathrm{C}$.

Figure 6 shows a $4 * 4$ Double Peres gate.

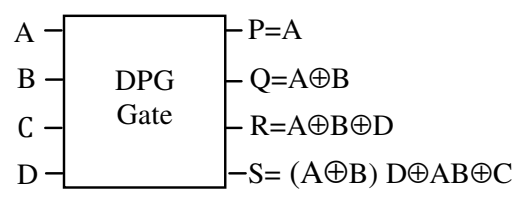

Figure 6: DPG gate 


\section{Related Work}

\subsection{RS Flip-Flop Designs}

Thapliyal [18] proposed reversible clocked $R S$ Flip-Flop which is a direct realization of conventional irreversible circuit realized. His design is costly because it incorporated two New gates whose quantum cost is higher than other reversible gates which is mentioned at Section 2 in Definition 2.4. Moreover, the design contained a single fan-out which is forbidden in strict reversible sense [23].Ashis [17] proposed design perform NAND operations using a single $3 * 3$ Peres gate instead of using Fredkin gate, his design less costly reversible $\bar{R} \bar{S}$ Flip-Flop using the Peres gate.Since the design of Thapliyal [18] consists of two New gates and the quantum cost of each New gate is 11, so the overall design cost increased. Moreover Ashis [17] realization contained two redundant Feynman gates. But Ashis [17] design is less costly in terms of number of gates, garbage bits and quantum costs than Thapliyal [18] design.

\subsection{Flip-Flop Designs}

Thapliyal [18] proposed a reversible clocked $D$ Flip-Flop that is also costly because it contained four New gates. Moreover a fanout can be seen in the design. Ashis [17] proposed design of reversible clocked $D$ Flip-Flop employs the proposed $\bar{R} \bar{S}$ latch. The clocked $D$ Flip-Flop is efficient to use in designing reversible memory circuits because of its superiority over the existing one [18] in terms of number of gates, garbage bits and quantum cost.

\section{Proposed Reversible Logic Gate}

We have proposed a new $4 * 4$ reversible logic gate named BME gate. The input vector is $\mathrm{I}(\mathrm{A}, \mathrm{B}, \mathrm{C}, \mathrm{D})$ and the output vector is $\mathrm{O}(\mathrm{P}, \mathrm{Q}, \mathrm{R}, \mathrm{S})$. The output is defined by $\mathrm{P}=\mathrm{A}, \mathrm{Q}=\mathrm{AB} \oplus \mathrm{C}$, $\mathrm{R}=\mathrm{AD} \oplus \mathrm{C}$ and $\mathrm{S}=\overline{\mathrm{A}} \mathrm{B} \oplus \mathrm{C} \oplus \mathrm{D}$. The block diagram of $\mathrm{BME}$ gate is shown in Figure 7 .

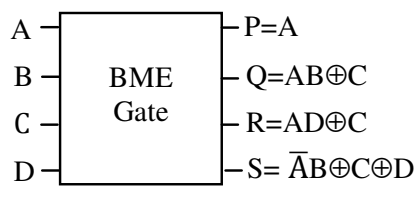

Figure 7: Block diagram of a new reversible BME gate

Table 1: Truth Table of New Reversible BME Gate

\begin{tabular}{|c|c|c|c|c|c|c|c|}
\hline $\mathrm{A}$ & $\mathrm{B}$ & $\mathrm{C}$ & $\mathrm{D}$ & $\mathrm{A}$ & $\mathrm{AB} \oplus \mathrm{C}$ & $\mathrm{AD} \oplus \mathrm{C}$ & $\overline{\mathrm{A}} \mathrm{B} \oplus \mathrm{C} \oplus \mathrm{D}$ \\
\hline 0 & 0 & 0 & 0 & 0 & 0 & 0 & 0 \\
\hline 0 & 0 & 0 & 1 & 0 & 0 & 0 & 1 \\
\hline 0 & 0 & 1 & 0 & 0 & 1 & 1 & 1 \\
\hline 0 & 0 & 1 & 1 & 0 & 1 & 1 & 0 \\
\hline 0 & 1 & 0 & 0 & 0 & 0 & 0 & 1 \\
\hline 0 & 1 & 0 & 1 & 0 & 0 & 0 & 0 \\
\hline 0 & 1 & 1 & 0 & 0 & 1 & 1 & 0 \\
\hline 0 & 1 & 1 & 1 & 0 & 1 & 1 & 1 \\
\hline 1 & 0 & 0 & 0 & 1 & 0 & 0 & 0 \\
\hline 1 & 0 & 0 & 1 & 1 & 0 & 1 & 1 \\
\hline 1 & 0 & 1 & 0 & 1 & 1 & 1 & 1 \\
\hline
\end{tabular}


International Journal of VLSI design \& Communication Systems (VLSICS) Vol.2, No.4, December 2011

\begin{tabular}{|l|l|l|l|l|l|l|l|}
\hline 1 & 0 & 1 & 1 & 1 & 1 & 0 & 0 \\
\hline 1 & 1 & 0 & 0 & 1 & 1 & 0 & 0 \\
\hline 1 & 1 & 0 & 1 & 1 & 1 & 1 & 1 \\
\hline 1 & 1 & 1 & 0 & 1 & 0 & 1 & 1 \\
\hline 1 & 1 & 1 & 1 & 1 & 0 & 0 & 0 \\
\hline
\end{tabular}

We can verify our BME gate by the truth table that the output and input vectors have one to one mapping between them which satisfies the condition of reversibility of a gate. We can see that from Table 1 that the 16 different input and output vectors are unique means they have one to one mapping between them. So, BME gate satisfies the condition of reversibility.

\section{Proposed Design of Reversible RS Flip-Flop}

The RS Flip-Flop can be mapped with one BME and two Peres gate. The BME gate needs E, S, 1 and $\mathrm{R}$ inputs respectively in $1^{\text {st }}, 2^{\text {nd }}, 3^{\text {rd }}$ and $4^{\text {th }}$ input. The output $\overline{E . S}$ and $\overline{E . R}$ are realized by one BME in the $2^{\text {nd }}$ and $3^{\text {rd }}$ outputs. Now, the $2^{\text {nd }}$ output of $\overline{E . S}$ that is $\bar{S}$ can be used as $2^{\text {nd }}$ input of one Peres gate. The $3^{\text {rd }}$ output of $\overline{E . R}$ that is $\overline{\mathrm{R}}$ can be used as $2^{\text {nd }}$ input of another Peres gate. Thus, our design needs only 3 reversible logic gates with 4 garbage outputs to design of RS FlipFlip. The proposed design of RS Flip-Flip is shown in Figure 8.

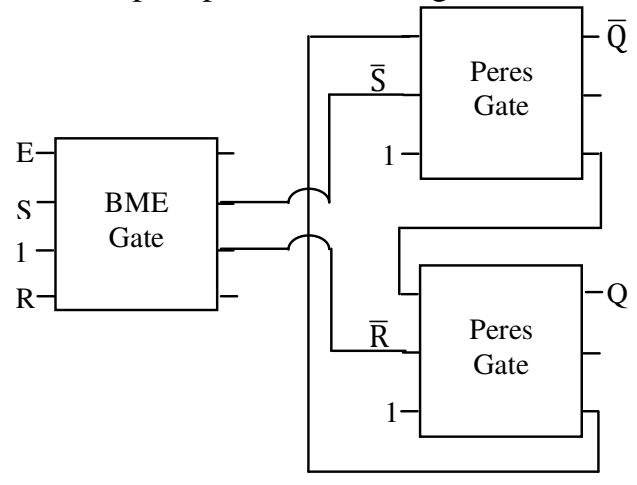

Figure 8: Proposed RS Flip-Flop

\subsection{Evaluation of Proposed RS Flip-Flop}

Table 2: Comparison of Different RS Flip-Flop

\begin{tabular}{|l|c|c|}
\hline & No of gates & No of garbage outputs \\
\hline Proposed design & 3 & 4 \\
\hline Existing design [17] & 5 & 6 \\
\hline Existing design [18] & 6 & 8 \\
\hline
\end{tabular}

From Table 2 we can see that the design is better than the design [17] and [18].Here, we need only 3 gates and 4 garbage outputs. So, the design is optimized than the design of [17] and [18] in terms of no of gates and garbage outputs. 


\section{Proposed Design of Reversible D Flip-Flop Using Proposed RS Flip- Flop}

The D Flip-Flop can be mapped with one BME and two Peres gate. The BME gate needs D, CLK, 1 and 0 inputs respectively in $1^{\text {st }}, 2^{\text {nd }}, 3^{\text {rd }}$ and $4^{\text {th }}$ input. The output $\overline{\mathrm{D} . \mathrm{CLK}}$ and $\overline{\overline{\mathrm{D}}}$. CLK are realized by one BME in the $2^{\text {nd }}$ and $4^{\text {th }}$ outputs. Now, the $2^{\text {nd }}$ output $\overline{\mathrm{D} . \mathrm{CLK}}$ that is $\bar{S}$ can be used as $2^{\text {nd }}$ input of one Peres gate. The $4^{\text {th }}$ output $\overline{\bar{D}}$. CLK that is $\bar{R}$ can be used as $2^{\text {nd }}$ input of another Peres gate. Thus, our design needs only 3 reversible logic gates with 4 garbage outputs to design of RS Flip-Flip. The proposed design of D Flip-Flip is shown in Figure 9.

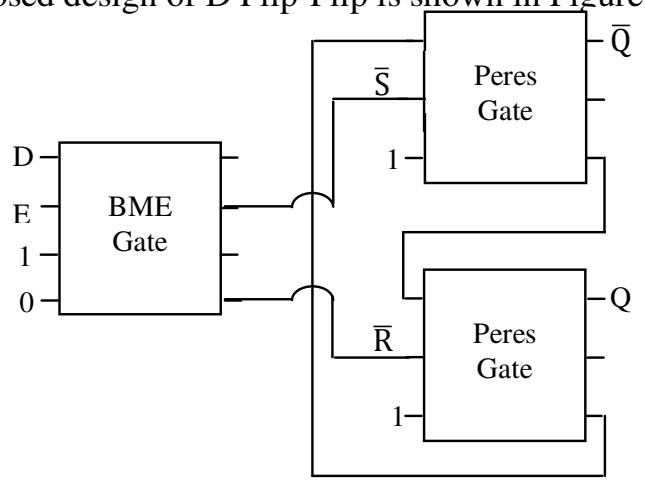

Figure 9: Proposed reversible clocked D Flip-Flop using Proposed RS Flip-Flop

\subsection{Evaluation of Proposed design of D Flip-Flop using Proposed RS Flip-Flop}

Table 3: Comparison of Different D Flip-Flop

\begin{tabular}{|l|c|c|}
\hline & No of gates & No of garbage outputs \\
\hline Proposed design & 3 & 4 \\
\hline Existing design [17] & 5 & 5 \\
\hline Existing design [18] & 7 & 8 \\
\hline
\end{tabular}

From Table 3 we can see that the design is better than the design [17] and [18].Here, we need only 3 gates and 4 garbage output. So, the design is optimized than the design of [17] and [18] in terms of no of gates and garbage outputs.

\section{Conclusions}

The proposed reversible design is utilized for efficiently designing RS and D Flip-Flop. As, FlipFlips are most important memory elements and used in several circuits like RAM, Logic Blocks of FPGA. We have seen by comparing the existing design with our proposed design that the proposed design is less costly in terms of number of gates and number of garbage outputs. The proposed design is highly optimized. So, this proposed gate can contribute significantly in the reversible logic community. Thus, the resulting reversible sequential circuits are more cost competent. 
International Journal of VLSI design \& Communication Systems (VLSICS) Vol.2, No.4, December 2011

\section{References}

[1] R. Landauer, "Irreversibility and heat generation in the computing process," IBM J. Research \& Development, vol. 5, no. 3, pp. 183-191,July 1961.

[2] C. Bennett, "Logical reversibility of computation," IBM J. Research \& Development, vol. 17, no. 6, pp. 525-532, Nov. 1973.

[3] S. Younis and T. Knight, "Asymptotically Zero Energy Split-Level Charge Recovery Logic," Workshop on Low Power Design, 1994.

[4] T. Toffoli, Automata, Languages and Programming. Springer Verlag, 1980, chapter: Reversible Computing, pp. 632-644.

[5] Babu HMH, Islam MR, Chowdhury AR, Chowdhury SMA. "Synthesis of full-adder circuit using reversible logic", 17th International Conference on VLSI Design 2004, 757-60.

[6] T. Toffoli., "Reversible Computing”, Tech memo MIT/LCS/TM-151, MIT Lab for Computer Science (1980).

[7] E. Fredkin and T. Toffoli, “Conservative logic,” Int'1 J. Theoretical Physics, Vol. 21, pp.219 253, 1982.

[8] H.R.Bhagyalakshmi and M.K.Venkatesha, "Optimized reversible BCD adder using new reversible logic gates", Journal of Computing, Volume 2, Issue 2, February 2010, ISSN 2151-9617 arXiv: $1002.3994 \mathrm{v} 1$.

[9] M. Haghparast,K. Navi, "A Novel Reversible BCD Adder For Nanotechnology Based Systems", American Journal of Applied Sciences 5 (3): 282-288, 2008,ISSN 1546-9239.

[10] Ashis Kumer Biswas, Md. Mahmudul Hasan, Moshaddek Hasan, Ahsan Raja Chowdhury and Hafiz Md. Hasan Babu. "A Novel Approach to Design BCD Adder and Carry Skip BCD Adder". 21st International Conference on VLSI Design, 1063-9667/08 $\$ 25.00$ (C) 2008 IEEE DOI 10.1109/VLSI.2008.37.

[11] H.R.Bhagyalakshmi, M.K.venkatesha. "An improved design of a multiplier using reversible logic gates”. International Journal of Engineering Science and Technology, Vol. 2(8), 2010, 3838-3845.

[12] Abu Sadat Md. Sayem, Masashi Ueda." Optimization of reversible sequential circuits". Journal of Computing, Volume 2, Issue 6, June 2010, ISSN 2151-9617.

[13] R. Feynman, “Quantum Mechanical Computers,” Optics News, Vol.11, pp. 11-20, 1985.

[14] B. Parhami, "Fault Tolerant Reversible Circuits" Proc. 40th Asilomar Conf. Signals, Systems, and Computers, Pacific Grove, CA, Oct.2006.

[15] A. Peres, "Reversible Logic and Quantum Computers", Physical review A, 32:3266- 3276, 1985.

[16] W. N. N. Hung, X. Song, G. Yang, J. Yang and M. Perkowski, "Quantum Logic Synthesis by Symbolic Reachability Analysis”, Proc. 41stannual conference on Design automation DAC, pp.838841, January 2004.

[17] Ashis Kumer Biswas, Lafifa Jamal, M. A. Mottalib1, Hafiz Md. Hasan Babu.” Design of a Reversible Parallel Loading Shift Register ".Dhaka Univ. J.Eng \& Tech. Vol 1(2) 1-5,2011.

[18] Himanshu Thapliyal, M.B.Srinivas, and M.Zwolinski, "A Beginning in the Reversible Logic Synthesis of Sequential Circuits “,MAPLD Conference (NASA office of Logic Design ), vol.8 2005. 
International Journal of VLSI design \& Communication Systems (VLSICS) Vol.2, No.4, December 2011

[19] J.Smoline and David P.DiVincenzo, "Five two-qubit gates are sufficient to implement the quantum fredkin gate", Physics Review A, vol. 53, no.4, pp. 2855-2856, 1996.

[20] Yang G., Song X, Hung WNN, and Marek Perkowski, "Bi-direction Synthesis for Reversible circuits”, IEEE Computer Society Annua; Symposium on VLSI New Frontiers in VLSI Design, 2007.

[21] D. Maslov and G.W. Dueck, "Reversible cascades with Minimal Garbage", IEEE Transactions on CAD, vol. 23(11), pp. 1497-1509, November 2004.

[22] Richard P.Feynman, "Quantum mechanical computers", Foundations of Physics, vol.16, no. 6, pp.507-531,1986.

[23] D. Maslov, G.W. Dueck, D.M. Miller, “Toffoli Network Synthesis with templates", IEEE Transactions on Computer Aided Design of Integrated Circuits and Systems, Vol.24, Issue 6, June 2005, pp:807-817

\section{Authors}

Md. Belayet Ali, received his B.Sc (Engg.) degree in Computer Science and Engineering from Mawlana Bhashani Science and Technology University, Santosh, Tangail-1902, Bangladesh, in 2008. He is working as Lecturer in Department of Computer Science and Engieering at (MBSTU), Santosh, Tangail-1902, Bangladesh. He has 2(two) years experience of working in MBSTU. He has published 04(four) papers in national, international journals. His area of interest includes reversible logic synthesis, multi-valued reversible logic synthesis, network business security, cloud computing. 